\title{
SUBCLASSES OF ANALYTIC FUNCTIONS WITH RESPECT TO SYMMETRIC AND CONJUGATE POINTS
}

\author{
C. SELVARAJ AND N. VASANTHI
}

Abstract. In this paper, we introduce new subclasses of convex and starlike functions with respect to other points. The coefficient estimates for these classes are obtained.

\section{Introduction}

Let $U$ be the class of functions which are analytic and univalent in the open unit disc $D=\{z:|z|<1\}$ given by

$$
w(z)=z+\sum_{k=1}^{n} b_{k} z^{k}
$$

and satisfying the conditions

$$
w(0)=0,|w(z)|<1, \quad z \in D .
$$

Let $S$ denote the class of functions $f$ which are analytic and univalent in $D$ of the form

$$
f(z)=z+\sum_{n=2}^{\infty} a_{n} z^{n}, \quad z \in D
$$

Also let $S_{s}^{*}$ be the subclass of $S$ consisting of functions given by (1.1) satisfying

$$
\operatorname{Re}\left\{\frac{z f^{\prime}(z)}{f(z)-f(-z)}\right\}>0, \quad z \in D
$$

These functions are called starlike with respect to symmetric points and were introduced by Sakaguchi in 1959. Ashwah and Thomas in [2] introduced another class namely the class $S_{c}^{*}$ consisting of functions starlike with respect to conjugate points.

Corresponding author: N. Vasanthi.

Received October 13, 2009.

2000 Mathematics Subject Classification. 30C45.

Key words and phrases. Analytic, univalent, starlike with respect to symmetric points, coefficient estimates. 
Let $S_{c}^{*}$ be the subclass of $S$ consisting of functions given by (1.1) and satisfying the condition

$$
\operatorname{Re}\left\{\frac{z f^{\prime}(z)}{f(z)+\overline{f(\bar{z})}}\right\}>0, \quad z \in D
$$

Motivated by $S_{s}^{*}$, many authors discussed the following class $C_{s}$ of function convex with respect to symmetric points and its subclasses.

Let $C_{s}$ be the subclass of $S$ consisting of functions given by (1.1) and satisfying the condition

$$
\operatorname{Re}\left\{\frac{\left(z f^{\prime}(z)\right)^{\prime}}{(f(z)-f(-z))^{\prime}}\right\}>0, \quad z \in D
$$

In terms of subordination, Goel and Mehrok in 1982 introduced a subclass of $S_{s}^{*}$ denoted by $S_{s}^{*}(A, B)$.

Let $S_{s}^{*}(A, B)$ be the class of functions of the form (1.1) and satisfying the condition

$$
\frac{2 z f^{\prime}(z)}{f(z)-f(-z)}<\frac{1+A z}{1+B z}, \quad-1 \leq B<A \leq 1, z \in D .
$$

Also let $S_{c}^{*}(A, B)$ be the class of functions of the form (1.1) and satisfying the condition

$$
\frac{2 z f^{\prime}(z)}{(f(z)+\overline{f(\bar{z})})}<\frac{1+A z}{1+B z}, \quad-1 \leq B<A \leq 1, z \in D .
$$

Let $C_{S}(A, B)$ be the class of functions of the form (1.1) and satisfying the condition

$$
\frac{2\left(z f^{\prime}(z)\right)^{\prime}}{(f(z)-f(-z))^{\prime}}<\frac{1+A z}{1+B z}, \quad-1 \leq B<A \leq 1, z \in D .
$$

Also let $C_{c}(A, B)$ be the class of functions of the form (1.1) and satisfying the condition

$$
\frac{2\left(z f^{\prime}(z)\right)^{\prime}}{(f(z)+\overline{f(\bar{z})})^{\prime}}<\frac{1+A z}{1+B z}, \quad-1 \leq B<A \leq 1, z \in D .
$$

In this paper, we introduce the class $M_{s}(\alpha, A, B)$ consisting of analytic functions $f$ of the form (1.1) and satisfying

$$
\begin{gathered}
\frac{2 z f^{\prime}(z)+2 \alpha z^{2} f^{\prime \prime}(z)}{(1-\alpha)(f(z)-f(-z))+\alpha z(f(z)-f(-z))^{\prime}}<\frac{1+A z}{1+B z}, \\
-1 \leq B<A \leq 1,0 \leq \alpha \leq 1, z \in D .
\end{gathered}
$$

We note that $M_{s}(0, A, B)=S_{s}^{*}(A, B)$ and $M_{s}(1, A, B)=C_{s}(A, B)$. Also introduce the class $M_{c}(\alpha, A, B)$ consisting of analytic functions $f$ of the form (1.1) and satisfying

$$
\begin{gathered}
\frac{2 z f^{\prime}(z)+2 \alpha z^{2} f^{\prime \prime}(z)}{(1-\alpha)(f(z)+\overline{f(\bar{z})})+\alpha z(f(z)+\overline{f(\bar{z}})^{\prime}}<\frac{1+A z}{1+B z} \\
-1 \leq B<A \leq 1,0 \leq \alpha \leq 1, z \in D .
\end{gathered}
$$


Note that $M_{c}(0, A, B)=S_{c}^{*}(A, B)$ and $M_{c}(1, A, B)=C_{c}(A, B)$.

By definition of subordination it follows that $f \in M_{s}(\alpha, A, B)$ if and only if

$$
\frac{2 z f^{\prime}(z)+2 \alpha z^{2} f^{\prime \prime}(z)}{(1-\alpha)(f(z)-f(-z))+\alpha z(f(z)-f(-z))^{\prime}}=\frac{1+A w(z)}{1+B w(z)}=p(z), \quad w \in U
$$

and that $f \in M_{c}(\alpha, A, B)$ if and only if

$$
\frac{2 z f^{\prime}(z)+2 \alpha z^{2} f^{\prime \prime}(z)}{(1-\alpha)(f(z)+\overline{f(\bar{z})})+\alpha z(f(z)+\overline{f(\bar{z})})^{\prime}}=\frac{1+A w(z)}{1+B w(z)}=p(z), \quad w \in U
$$

where

$$
p(z)=1+\sum_{n=1}^{\infty} p_{n} z^{n}
$$

We study the classes $M_{s}(\alpha, A, B)$ and $M_{c}(\alpha, A, B)$, the coefficient estimates for functions belonging to these classes are obtained.

\section{Preliminary result}

We need the following lemma for proving our results.

Lemma 2.1.([3]) If $p(z)$ is given by (1.4) then

$$
\left|p_{n}\right| \leq A-B, \quad n=1,2,3, \ldots
$$

\section{Main result}

We give the coefficient inequalities for the classes $M_{\mathcal{S}}(\alpha, A, B)$ and $M_{c}(\alpha, A, B)$.

Theorem 3.1. Let $f \in M_{s}(\alpha, A, B)$. Then for $n \geq 1,0 \leq \alpha \leq 1$,

$$
\begin{aligned}
\left|a_{2 n}\right| & \leq \frac{A-B}{2^{n} n !(1+(2 n-1) \alpha)} \prod_{j=1}^{n-1}(A-B+2 j), \\
\left|a_{2 n+1}\right| & \leq \frac{A-B}{2^{n} n !(1+2 n \alpha)} \prod_{j=1}^{n-1}(A-B+2 j) .
\end{aligned}
$$

Proof. From (1.2) and (1.4), we have

$$
\begin{aligned}
\left(z+2 a_{2} z^{2}+3 a_{3} z^{3}+4 a_{4} z^{4}+5 a_{5} z^{5}+\cdots+2 n a_{2 n} z^{2 n}+\cdots\right) \\
+\alpha\left(2 a_{2} z^{2}+6 a_{3} z^{3}+12 a_{4} z^{4}+20 a_{5} z^{5}+\cdots+(2 n-1) 2 n a_{2 n} z^{2 n}+\cdots\right) \\
=\left[(1-\alpha)\left(z+a_{3} z^{3}+a_{5} z^{5}+\cdots+a_{2 n-1} z^{2 n-1}+a_{2 n+1} z^{2 n+1}+\cdots\right)\right.
\end{aligned}
$$




$$
\begin{aligned}
& \left.+\alpha\left(z+3 a_{3} z^{3}+5 a_{5} z^{5}+\cdots+(2 n-1) a_{2 n-1} z^{2 n-1}+(2 n+1) a_{2 n+1} z^{2 n+1}+\cdots\right)\right] \\
& \cdot\left(1+p_{1} z+p_{2} z^{2}+p_{3} z^{3}+p_{4} z^{4}+\cdots+p_{2 n-1} z^{2 n-1}+p_{2 n} z^{2 n}+\cdots\right)
\end{aligned}
$$

Equating the coefficients of like powers of $z$, we have

$$
\begin{aligned}
2(1+\alpha) a_{2} & =p_{1}, 2(1+2 \alpha) a_{3}=p_{2} \\
4(1+3 \alpha) a_{4} & =p_{3}+(1+2 \alpha) a_{3} p_{1} \\
4(1+4 \alpha) a_{5} & =p_{4}+(1+2 \alpha) a_{3} p_{2} \\
2 n(1+(2 n-1) \alpha) a_{2 n} & =p_{2 n-1}+(1+2 \alpha) a_{3} p_{2 n-3}+\cdots+(1+(2 n-2) \alpha) a_{2 n-1} p_{1} \\
(2 n+1)(1+2 n \alpha) a_{2 n+1} & =p_{2 n}+(1+2 \alpha) a_{3} p_{2 n-2}+\cdots+(1+(2 n-2) \alpha) a_{2 n-1} p_{2}
\end{aligned}
$$

Using lemma 2.1 and (3.3), we get

$$
\left|a_{2}\right| \leq \frac{A-B}{2(1+\alpha)}, \quad\left|a_{3}\right| \leq \frac{A-B}{2(1+2 \alpha)} .
$$

Again by applying (3.6) and followed by Lemma 2.1, we get from (3.4)

$$
\left|a_{4}\right| \leq \frac{(A-B)(A-B+2)}{(2)(4)(1+3 \alpha)}, \quad\left|a_{5}\right| \leq \frac{(A-B)(A-B+2)}{(2)(4)(1+4 \alpha)} .
$$

It follows that (3.1) and (3.2) hold for $n=1,2$. We prove (3.1) using induction.

Equation (3.5) in conjuction with lemma 2.1 yield

$$
\left|a_{2 n}\right| \leq \frac{A-B}{2 n(1+(2 n-1) \alpha)}\left[1+\sum_{k=1}^{n-1}(1+2 k \alpha)\left|a_{2 k+1}\right|\right] .
$$

We assume that (3.1) holds for $k=3,4, \ldots,(n-1)$. Then from (3.8), we obtain

$$
\left|a_{2 n}\right| \leq \frac{A-B}{2 n(1+(2 n-1) \alpha)}\left[1+\sum_{k=1}^{n-1} \frac{A-B}{2^{k} k !} \prod_{j=1}^{k-1}(A-B+2 j)\right] .
$$

In order to complete the proof, it is sufficient to show that

$$
\begin{aligned}
& \frac{A-B}{2 m(1+(2 m-1) \alpha)}\left[1+\sum_{k=1}^{m-1} \frac{A-B}{2^{k} k !} \prod_{j=1}^{k-1}(A-B+2 j)\right] \\
& =\frac{A-B}{2^{m} m !(1+(2 m-1) \alpha)} \prod_{j=1}^{k-1}(A-B+2 j), \quad(m=3,4,5, \ldots, n) .
\end{aligned}
$$

(3.10) is valid for $m=3$.

Let us suppose that (3.10) is true for all $m, 3<m \leq(n-1)$. Then from (3.9)

$$
\frac{(A-B)}{2 n(1+(2 n-1) \alpha)}\left[1+\sum_{k=1}^{n-1} \frac{A-B}{2^{k} k !} \prod_{j=1}^{k-1}(A-B+2 j)\right]
$$




$$
\begin{aligned}
= & \left(\frac{n-1}{n}\right)\left(\frac{A-B}{2(n-1)(1+(2 n-1) \alpha)}\left(1+\sum_{k=1}^{n-2} \frac{A-B}{2^{k} k !} \prod_{j=1}^{k-1}(A-B+2 j)\right)\right) \\
& +\frac{(A-B)}{(2 n)(1+(2 n-1) \alpha)} \frac{(A-B)}{(n-1) ! 2^{n-1}} \prod_{j=1}^{n-2}(A-B+2 j) \\
= & \left(\frac{n-1}{n}\right) \frac{(A-B)}{2^{n-1}(n-1) !(1+(2 n-1) \alpha)} \prod_{j=1}^{n-2}(A-B+2 j) \\
& +\frac{(A-B)}{2 n(1+(2 n-1) \alpha)} \frac{(A-B)}{(n-1) ! 2^{n-1}} \prod_{j=1}^{n-2}(A-B+2 j) \\
= & \frac{(A-B)}{2 n(n-1) ! 2^{n-1}(1+(2 n-1) \alpha)} \prod_{j=1}^{n-2}(A-B+2 j)(A-B+2(n-1)) \\
= & \frac{(A-B)}{2^{n} n !(1+(2 n-1) \alpha)} \prod_{j=1}^{n-1}(A-B+2 j) .
\end{aligned}
$$

Thus (3.10) holds for $m=n$ and hence (3.1) follows. Similarly we can prove (3.2).

Theorem 3.2. Let $f \in M_{c}(\alpha, A, B)$. Then for $n \geq 1,0 \leq \alpha \leq 1$,

$$
\begin{aligned}
\left|a_{2 n}\right| & \leq \frac{(A-B)}{(2 n-1) !(1+(2 n-1) \alpha)} \prod_{j=1}^{2 n-2}(A-B+j), \\
\left|a_{2 n+1}\right| & \leq \frac{(A-B)}{(2 n) !(1+2 n \alpha)} \prod_{j=1}^{2 n-1}(A-B+j) .
\end{aligned}
$$

Proof. From (1.3) and (1.4), we have

$$
\begin{aligned}
(z+ & \left.+2 a_{2} z^{2}+3 a_{3} z^{3}+4 a_{4} z^{4}+5 a_{5} z^{5}+\cdots+2 n a_{2 n} z^{2 n}+\cdots\right) \\
& +\alpha\left(2 a_{2} z^{2}+6 a_{3} z^{3}+12 a_{4} z^{4}+20 a_{5} z^{5}+\cdots+(2 n-1) 2 n a_{2 n} z^{2 n}+\cdots\right) \\
= & {\left[(1-\alpha)\left(z+a_{2} z^{2}+a_{3} z^{3}+a_{4} z^{4}+a_{5} z^{5}+\cdots+a_{2 n} z^{2 n}+\cdots\right)\right.} \\
& \left.+\alpha\left(z+2 a_{2} z^{2}+3 a_{3} z^{3}+4 a_{4} z^{4}+5 a_{5} z^{5}+\cdots+2 n a_{2 n} z^{2 n}+\cdots\right)\right] \\
& \cdot\left(1+p_{1} z+p_{2} z^{2}+p_{3} z^{3}+p_{4} z^{4}+\cdots+p_{2 n-1} z^{2 n-1}+\cdots\right)
\end{aligned}
$$

Equating the coefficients of like powers of $z$, we have

$$
\begin{aligned}
(1+\alpha) a_{2} & =p_{1}, 2(1+2 \alpha) a_{3}=p_{2}+(1+\alpha) a_{2} p_{1} \\
3(1+3 \alpha) a_{4} & =p_{3}+(1+\alpha) a_{2} p_{2}+(1+2 \alpha) a_{3} p_{1} \\
4(1+4 \alpha) a_{5} & =p_{4}+(1+\alpha) a_{2} p_{3}+(1+2 \alpha) a_{3} p_{2}+(1+3 \alpha) a_{4} p_{1} \\
(2 n-1)(1+(2 n-1) \alpha) a_{2 n} & =p_{2 n-1}+(1+\alpha) a_{2} p_{2 n-2}+\cdots+(1+(2 n-2) \alpha) a_{2 n-1} p_{1} \\
2 n(1+2 n \alpha) a_{2 n+1} & =p_{2 n}+(1+\alpha) a_{2} p_{2 n-1}+\cdots+(1+(2 n-2) \alpha) a_{2 n} p_{1}
\end{aligned}
$$


By using lemma 2.1 and (3.13), we get

$$
\left|a_{2}\right| \leq \frac{(A-B)}{1+\alpha}, \quad\left|a_{3}\right| \leq \frac{(A-B)(A-B+1)}{2(1+2 \alpha)} .
$$

Again by applying (3.18) and followed by Lemma 2.1, we get from (3.14) and (3.15), we have

$$
\begin{aligned}
& \left|a_{4}\right| \leq \frac{(A-B)(A-B+1)(A-B+2)}{(2)(3)(1+3 \alpha)}, \\
& \left|a_{5}\right| \leq \frac{(A-B)^{2}+6(A-B)^{3}+11(A-B)^{2}+6(A-B)}{(2)(3)(4)(1+4 \alpha)} .
\end{aligned}
$$

It follows that (3.11) hold for $n=1,2$. We now prove (3.11) using induction.

Equation (3.16) in conjuction with lemma 2.1 yield

$$
\left|a_{2 n}\right| \leq \frac{(A-B)}{(2 n-1)(1+(2 n-1) \alpha)}\left[1+\sum_{k=1}^{n-1}\left|a_{2 k}\right|+\sum_{k=1}^{n-1}\left|a_{2 k+1}\right|\right] .
$$

We assume that (3.11) holds for $k=3,4, \ldots,(n-1)$. Then from (3.19), we obtain

$$
\begin{aligned}
\left|a_{2 n}\right| \leq & \frac{(A-B)}{(2 n-1)(1+(2 n-1) \alpha)}\left[1+\sum_{k=1}^{n-1} \frac{(A-B)}{(2 k-1) !} \prod_{j=1}^{2 k-2}(A-B+j)\right. \\
& \left.+\sum_{k=1}^{n-1} \frac{(A-B)}{(2 k) !} \prod_{j=1}^{2 k-1}(A-B+j)\right] .
\end{aligned}
$$

In order to complete the proof, it is sufficient to show that

$$
\begin{gathered}
\frac{(A-B)}{(2 m-1)(1+(2 m-1) \alpha)}\left[1+\sum_{k=1}^{m-1} \frac{(A-B)}{2(k-1) !} \prod_{j=1}^{2 k-2}(A-B+j)+\sum_{k=1}^{m-1} \frac{(A-B)}{(2 k) !} \prod_{j=1}^{2 k-1}(A-B+j)\right] \\
=\frac{(A-B)}{(2 m-1) !(1+(2 m-1) \alpha)} \prod_{j=1}^{2 m-2}(A-B+j), \quad(m=3,4,5, \ldots, n) .
\end{gathered}
$$

(3.21) is valid for $m=3$.

Let us suppose that (3.21) is true for all $m, 3<m \leq(n-1)$. Then from (3.20)

$$
\begin{gathered}
\frac{(A-B)}{(2 n-1)(1+(2 n-1) \alpha)}\left[1+\sum_{k=1}^{n-1} \frac{(A-B)}{(2 k-1) !} \prod_{j=1}^{2 k-2}(A-B+j)+\sum_{k=1}^{n-1} \frac{(A-B)}{(2 k) !} \prod_{j=1}^{2 k-1}(A-B+j)\right] \\
=\frac{(2 n-3)}{(2 n-1)}\left(\frac { ( A - B ) } { ( 2 ( n - 1 ) - 1 ) ( 1 + ( 2 n - 1 ) \alpha ) } \left(1+\sum_{k=1}^{n-2} \frac{(A-B)}{(2 k-1) !} \prod_{j=1}^{2 k-2}(A-B+j)\right.\right. \\
\left.\left.+\sum_{k=1}^{n-2} \frac{(A-B)}{(2 k) !} \prod_{j=1}^{2 k-1}(A-B+j)\right)\right) \\
+\frac{(A-B)}{(2 n-1)(1+(2 n-1) \alpha)} \frac{(A-B)}{(2(n-1) !-1)} \prod_{j=1}^{2 n-4}(A-B+j)
\end{gathered}
$$




$$
\begin{aligned}
& +\frac{(A-B)}{(2 n-1)(1+(2 n-1) \alpha)} \frac{(A-B)}{2(n-1) !} \prod_{j=1}^{2 n-3}(A-B+j) \\
= & \frac{2 n-3}{(2 n-1)(1+(2 n-1) \alpha)} \frac{(A-B)}{(2(n-1)-1) !} \prod_{j=1}^{2 n-4}(A-B+j) \\
& +\frac{(A-B)}{(2 n-1)(1+(2 n-1) \alpha)} \frac{(A-B)}{(2(n-1)-1) !} \prod_{j=1}^{2 n-4}(A-B+j) \\
= & \frac{(A-B)}{(2 n-1)(1+(2 n-1) \alpha)(2(n-1)-1) !} \prod_{j=1}^{(2 n-1)(1+(2 n-1) \alpha)} \frac{(A-B)}{2(n-1) !} \prod_{j=1}^{2 n-3}(A-B+j) \\
& +\frac{(A-B)}{(2 n-1)(1+(2 n-1) \alpha)} \frac{(A-B)}{(2(n-1)) !} \prod_{j=1}^{2 n-3}(A-B+j) \\
= & \frac{(A-B)}{(2 n-1) !(1+(2 n-1) \alpha)} \prod_{j=1}^{2 n-2}(A-B+j) .
\end{aligned}
$$

Thus (3.21) holds for $m=n$ and hence (3.11) follows. Similarly we can prove (3.12).

On specializing the values of $\alpha$ in Theorem 3.1 and Theorem 3.2, we get the following.

Remark 3.1 . In Theorem 3.1, if we set $\alpha=0$, we get starlike functions with respect to symmetric points and if we set $\alpha=1$, we get convex functions with respect to symmetric points.

Remark 3.2. In Theorem 3.2, if we set $\alpha=0$, we get starlike functions with respect to conjugate points and if we set $\alpha=1$, we get convex functions with respect to conjugate points. For other values of $\alpha$ the transition is smooth.

\section{References}

[1] R. N. Das and P. Singh, On subclasses of schlicht mapping, Indian J. Pure Appl. Math., 8 (1977), 864-872.

[2] R. M. El-Ashwah and D. K. Thomas, Some subclasses of close-to-convex functions, J. Ramanujan Math. Soc., 2 (1987), 86-100.

[3] R. M. Goel and B. C. Mehrok, A subclass of starlike functions with respect to symmetric points, Tamkang J. Math., 13(1) (1982), 11-24.

[4] A. Janteng and S. A. F. M. Dahhar, A subclass of starlike functions with respect to conjugate points, Int. Mathematical Forum, 4 (2009), 1373-1377.

[5] A. Janteng and S. A. Halim, A subclass Quasi-convex functions with respect to symmetric points, Applied Mathematical Sciences, 3(2009), 551-556.

[6] A. Janteng and S. A. Halim, Coefficient estimates for a subclass of close-to-convex functions with respect to symmetric points, Int. J. Math. Analysis, 3(2009), 309-313.

[7] K. Sakaguchi, On certain univalent mapping, J. Math. Soc., Japan, 11 (1959), 72-75. 
[8] C. Selvaraj and K. A. Selvakumaran, Fekete-Szegö problem for some subclasses of analytic functions, Far East Journal of Mathematical Sciences, 29(2008), 643-652.

[9] C. Selvaraj and N. Vasanthi, A subclass of $\alpha$-Quasi-convex functions with respect to symmetric points, submitted.

Department of Mathematics, Presidency College, Chennai - 600 005, Tamil Nadu, India.

E-mail: pamc9439@yahoo.co.in

Department of Mathematics, Presidency College, Chennai - 600 005, Tamil Nadu, India.

E-mail: svvsaagar@yahoo.co.in 